\title{
What is rheumatoid arthritis? Considering consequences of changed classification criteria
}

\author{
Annette H M van der Helm-van Mil, ${ }^{1,2}$ Angela Zink ${ }^{3,4}$
}

\section{CHANGES IN CLASSIFICATION CRITERIA}

Rheumatoid arthritis (RA) was recognised as a separate disease entity in the middle of the 20th century. Since then several sets of classification criteria were developed for use in clinical studies. The 1987 American College of Rheumatology (ACR) criteria $^{1}$ have been criticised as they are fulfilled rather late in the disease. Consequently, trials that included patients with RA based on the 1987 criteria studied patients with relatively longstanding arthritis. The aim of the American College of Rheumatology/ European League Against Rheumatism (ACR/EULAR) expert panel was therefore to develop novel criteria without the features of long-standing disease such as erosions or nodules. Indeed the hitherto developed 2010 ACR/EULAR classification criteria for $\mathrm{RA}^{2}$ are fulfilled earlier in the disease course than the 1987 criteria. $^{3}{ }^{4}$ Thus, the major objective of the new criteria was achieved.

The study of Nordberg et $a l^{5}$ makes us think about the consequences of the novel criteria for the phenotype of RA. According to the 1987 criteria, the characteristic phenotype of RA consists of symmetric polyarthritis of small joints with morning stiffness (ignoring the features of long-standing disease nodules and erosions). Symmetry and morning stiffness are no longer included in the 2010 criteria. There is less emphasis on clinical elements in the 2010 criteria, and this is paralleled by an emphasis on the results of additional investigations: anti-citrullinated peptide antibodies (ACPA) and acute phase responses were

\footnotetext{
${ }^{1}$ Department of Rheumatology, Leiden University Medical Center, Leiden, The Netherlands; ' ${ }^{2}$ Department of Rheumatology, Erasmus Medical Center, Rotterdam, The Netherlands; ${ }^{3}$ Epidemiology Unit, German Rheumatism Research Centre, Berlin, Germany; ${ }^{4}$ Department for Rheumatology and Clinical Immunology, Charité University Medicine, Berlin Germany
}

Correspondence to Professor Dr Annette $\mathrm{H}$ M van der Helm-van Mil, Department of Rheumatology, Leiden University Medical Center, P.O. Box 9600, RC Leiden 2300, The Netherlands; AvdHelm@lumc.nl introduced (table 1). The majority of the six points required to fulfil the 2010 criteria can be achieved either by the presence of autoantibodies or on the number of swollen joints. Nordberg et al showed that patients with ACPA-positive RA fulfilling the 2010 criteria have less inflamed joints (measured with either physical examination or with ultrasound) than patients with ACPA-negative RA. This finding is not surprising if we consider the composition of the 2010 criteria.

For instance, a patient who presents with clinically apparent arthritis of nine joints, symmetrically distributed in both hands, with morning stiffness and in whom the symptoms persist for more than 6 weeks, has the classic presentation of RA as recognised by fulfilling the 1987 criteria for RA. According to 2010 criteria, this patient is not classified as having RA if there are no RA-related autoantibodies, even not if the acute phase response is elevated. In contrast, a patient who presents with one swollen joint, a high positive rheumatoid factor (RF) and a symptom duration of $>6$ weeks without an increased acute phase response is classified as RA according to the 2010 criteria. Although the issue that if certain characteristics are absent, others should be available to fulfil classification criteria is generic and not unique for RA, the descriptions of these two patients illustrate the imbalance between the extents of inflamed joints needed to classify RA according to both sets of criteria.

\section{CHANGES IN TEST CHARACTERISTICS}

What are the potential consequences of using the 2010 criteria? A systematic review of the performance of the 2010 criteria has shown that these criteria are more sensitive but less specific than the 1987 criteria. $^{6}$ Burgers et $a l^{7}$ reported a milder disease outcome in patients fulfilling the 2010 criteria than in those fulfilling the 1987 criteria. This finding is in line with the lower specificity and suggests that the 2010 criteria include patients who were formerly not indicated as RA and who have a better disease outcome. ${ }^{8}$

Despite the lower specificity, the advantage of the 2010 criteria is the higher sensitivity. Although it is not directly evaluated in the study of Nordberg et al, ${ }^{5}$ the results of this study suggest that the increase in sensitivity is largely confined to patients who are ACPA positive because patients who are ACPA negative now require more inflamed/involved joints to be classified as RA than those who are ACPA positive. Further studies are needed to verify this.

Patients who were classified as RA according to the 1987 criteria but not according to the 2010 criteria are largely ACPA negative; ${ }^{7}$ these patients were not studied by Norberg et al and will also be excluded from other studies on RA that are based on the 2010 criteria.

\section{CONSEQUENCES FOR CLINICAL \\ TRIALS}

In addition to the changes in test characteristics, another consequence of the changed criteria is that the general concept of $R A$ is shifting. Currently some clinicians or researchers consider the presence of autoantibodies the central hallmark of RA, rather than the clinical presentation. In other words, some consider patients who have no ACPA or no $\mathrm{RF}$ as not having RA. This underestimates the burden of seronegative RA, which is often recognised later and has been shown to be associated with greater unmet need of treatment than seropositive RA. ${ }^{8}$

Furthermore, if the 2010 criteria are used to classify RA and fulfilling the 2010 criteria is an inclusion criterion, these trials will include more patients with ACPA-positive RA than ACPA-negative RA. This may be illustrated by the study of Nordberg that showed that only $15 \%$ of the patients with 2010-criteria RA were ACPA negative and 85\% ACPA positive. A similar distribution was observed in data of the Canadian Early Arthritis Cohort (CATCH) study. ${ }^{9}$ Of note, other studies that compared both criteria did not observe this high prevalence of ACPA positivity when using the 2010 criteria; the percentage of ACPA-positive 2010-RA patients was $52 \%, 66 \%$ and $59 \%$ in these studies. $^{3} 710$

Currently, there are very few studies that used the 2010 criteria to include patients. $^{11}$ C-OPERA enrolled patients fulfilling the 2010 criteria, but they also needed to have poor prognostic factors, and all patients included were ACPA positive. ${ }^{12}$ A task force of European Society for Clinical and Economic Aspects of Osteoporosis, Osteoarthritis and Musculoskeletal Diseases (ESCEO) recommended recently that more trials should be done on early RA, as defined by the 2010 criteria. $^{13}$ Hence, future trials might 


\begin{tabular}{|c|c|}
\hline \multirow[t]{2}{*}{$\begin{array}{l}1987 \text { classification criteria } \\
\text { (parameters) }\end{array}$} & $\begin{array}{l}2010 \text { classification criteria } \\
\text { (parameters and points) }\end{array}$ \\
\hline & $\begin{array}{l}\text { To be used in patients with at least one joint with definite clinical } \\
\text { synovitis and with the synovitis not better explained by another disease }\end{array}$ \\
\hline Morning stiffness & $\begin{array}{l}\text { Joint involvement } \\
1 \text { large joint }=0 \\
2-10 \text { large joints }=1 \\
1-3 \text { small joints }=2 \\
4-10 \text { small joints }=3 \\
>10 \text { small joints }=5\end{array}$ \\
\hline Arthritis of three or more joint areas & $\begin{array}{l}\text { Serology } \\
\text { RF and ACPA negative }=0 \\
\text { Low positive RF or } A C P A=2 \\
\text { High positive RF or } A C P A=3\end{array}$ \\
\hline Arthritis of hand joints & $\begin{array}{l}\text { Acute phase reactants } \\
\text { Normal }=0 \\
\text { Elevated CRP or } E S R=1\end{array}$ \\
\hline Symmetric arthritis & $\begin{array}{l}\text { Duration of symptoms } \\
<6 \text { weeks }=0 \\
>6 \text { weeks }=1\end{array}$ \\
\hline \multicolumn{2}{|l|}{ Rheumatoid nodules } \\
\hline \multicolumn{2}{|l|}{ Serum RF } \\
\hline \multicolumn{2}{|l|}{ Radiographic changes } \\
\hline $\begin{array}{l}\text { Criteria are fulfilled if } 4 \text { out of } 7 \\
\text { parameters are present }\end{array}$ & Criteria are fulfilled if a patient has $\geq 6$ points \\
\hline
\end{tabular}

ACPA, anti-citrullinated peptide antibody; ACR, American College of Rheumatology; CRP, C-reactive protein; ESR, erythrocyte sedimentation rate; EULAR, European League Against Rheumatism; RA, rheumatoid arthritis; RF, rheumatoid factor.

be more focused on early ACPA-positive RA than on early ACPA-negative RA. This recommendation might further shift the evidence towards ACPA-positive disease.

\section{IS A FOCUS TOWARDS PATIENTS WITH ACPA-POSITIVE RA IN CLINICAL TRIALS A PROBLEM?}

ACPA-negative RA is a chronic disease. Patients with ACPA-negative RA require disease modifying anti-rheumatic drugs (DMARDs), and published data demonstrate that patients who are ACPA negative respond to common treatment strategies with methotrexate. ${ }^{14}{ }^{15}$ As for patients who are ACPA positive, also in patients who are ACPA negative early treatment is associated with a milder disease course than delayed treatment. ${ }^{14} 1617$ Altogether these results underline that patients who are ACPA negative deserve to be identified and treated early in time. An underrepresentation of patients who are ACPA negative in future clinical trials will hamper evidence-based treatment decisions in these patients. Furthermore, at the derivation of the 2010 criteria, it has not been decided that the presence of ACPA is mandatory and it was not decided to make separate criteria for patients who are ACPA positive and those who are ACPA negative; therefore the challenge now is to come with a solution for the patients who are ACPA negative.

\section{HOW TO SOLVE THE PROBLEM OF THE} PATIENTS WITH ACPA-NEGATIVE RA?

Without doubt, identification of ACPA-negative RA is more challenging than that of ACPA-positive RA, and the risk of false positive misclassification needs to be addressed. How to solve this issue? First, patients who are ACPA negative and who fulfilled the 1987 criteria but are 2010-criteria negative can still be considered to have RA, as they have either the characteristic phenotypic presentation of a chronic symmetric polyarthritis of small joins with morning stiffness, or they have features of long-standing disease such as erosions. However, also in ACPA-negative RA, the 1987 criteria may be fulfilled rather late in the disease and the question how to identify these patients early in the disease, and to differentiate them from other patients with ACPA negative arthritis, is still unsolved. ${ }^{18} 19$ Additional diagnostic tools are required and some are discussed below.

Novel RA-related autoantibodies have been identified, including antibodies against carbamylated or acetylated proteins, or antibodies directed against other (unpublished) proteins. Unfortunately, however, these novel autoantibodies largely overlap with the presence of ACPA and RF and they fill only a part of the so-called 'serological gap'. ${ }^{20-22}$
Imaging tools are currently been explored. Some initial studies suggested that MRI can significantly improve the diagnostic performance of the 2010 ACR/ EULAR classification criteria. ${ }^{23}$ A large recent study demonstrated that hand and foot MRI was particularly predictive in patients who were ACPA negative and presented with oligoarthritis. ${ }^{24}$ Although studies on ultrasound are more difficult to standardise and results might be evaluatordependent, there are data suggesting that ultrasound might also be of help in identifying ACPA-negative RA. ${ }^{25}$ Thus far, convincing evidence on the additive value of novel imaging modalities in clinical practice is lacking. Larger studies in patients who are ACPA negative are needed.

Thus, the 2010 criteria put more emphasis on the value of additional investigations than the 1987 criteria and ACPA is a powerful criterion. However, a diagnostic tool for ACPA-negative RA that has a similar value is still lacking.

\section{RESEARCH AGENDA}

In summary, the study of Nordberg et al points to an important phenomenon, but also leaves several questions unanswered. First, the results of Nordberg et al imply that the 2010 criteria perform less well in the identification of ACPA-negative RA than that of ACPA-positive RA; this presumption should be confirmed with data. Subsequently, novel tools are needed to identify patients with ACPA-negative RA early. Tools that detect inflammation sensitively might be promising and large studies performed in unselected sets of patients who are ACPA-negative, resembling the patients seen in daily practice, are urgently needed.

\section{Competing interests None declared.}

Provenance and peer review Commissioned; externally peer reviewed.

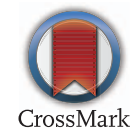

To cite van der Helm-van Mil AHM, Zink A. Ann Rheum Dis 2017;76:315-317.

Received 29 May 2016

Revised 1 September 2016

Accepted 4 September 2016

Published Online First 22 September 2016

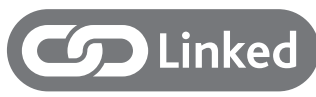

- http://dx.doi.org/10.1136/annrheumdis-2015208873

Ann Rheum Dis 2017:76:315-317.

doi:10.1136/annrheumdis-2016-209629 


\section{REFERENCES}

1 Arnett FC, Edworthy SM, Bloch DA, et al. The American Rheumatism Association 1987 revised criteria for the classification of rheumatoid arthritis. Arthritis Rheum 1988;31:315-24.

2 Aletaha D, Neogi T, Silman AJ, et al. 2010 rheumatoid arthritis classification criteria: an American College of Rheumatology/European League Against Rheumatism collaborative initiative. Ann Rheum Dis 2010;69:1580-8.

3 Cader MZ, Filer A, Hazlehurst J, et al. Performance of the 2010 ACR/EULAR criteria for rheumatoid arthritis: comparison with 1987 ACR criteria in a very early synovitis cohort. Ann Rheum Dis 2011:70:949-55.

4 van der Linden MP, Knevel R, Huizinga TW, et al. Classification of rheumatoid arthritis: comparison of the 1987 American College of Rheumatology criteria and the 2010 American College of Rheumatology/ European League Against Rheumatism criteria. Arthritis Rheum 2011;63:37-42.

5 Nordberg LB, Lillegraven S, Lie E, et al. Patients with seronegative RA have more inflammatory activity compared with patients with seropositive RA in an inception cohort of DMARD-naive patients classified according to the 2010 ACR/EULAR criteria. Ann Rheum Dis 2017;76:341-5.

6 Radner H, Neogi T, Smolen JS, et al. Performance of the 2010 ACR/EULAR classification criteria for rheumatoid arthritis: a systematic literature review. Ann Rheum Dis 2014:73:114-23.

7 Burgers $L E$, van Nies JA, Ho LY, et al. Long-term outcome of rheumatoid arthritis defined according to the 2010-classification criteria. Ann Rheum Dis 2014;73:428-32.

8 Westhoff G, Schneider M, Raspe HH, et al. Advance and unmet need of health care for patients with rheumatoid arthritis in the German population--results from the German Rheumatoid Arthritis Population Survey (GRAPS). Rheumatology (Oxford) 2009;48:650-7.

9 Shu J, Bykerk VP, Boire G, et al. Missing anticitrullinated protein antibody does not affect short-term outcomes in early inflammatory arthritis: from the Canadian Early Arthritis Cohort. J Rheumatol 2015:42:2023-8.

10 Britsemmer K, Ursum J, Gerritsen M, et al. Validation of the 2010 ACR/EULAR classification criteria for rheumatoid arthritis: slight improvement over the 1987 ACR criteria. Ann Rheum Dis 2011;70: 1468-70. Erratum in: Ann Rheum Dis 2011;70: 2060. van Tuyl $L$ (corrected to van Tuyl, LH).

11 de Jong PH, Hazes JM, Han HK, et al. Randomised comparison of initial triple DMARD therapy with methotrexate monotherapy in combination with low-dose glucocorticoid bridging therapy; 1-year data of the tREACH trial. Ann Rheum Dis 2014;73: 1331-9.

12 Atsumi T, Yamamoto K, Takeuchi T, et al. The first double-blind, randomised, parallel-group certolizumab pegol study in methotrexate-naive early rheumatoid arthritis patients with poor prognostic factors, C-OPERA, shows inhibition of radiographic progression. Ann Rheum Dis 2016;75:75-83.

13 Smolen JS, Collaud BS, Boers M, et al. Clinical trials of new drugs for the treatment of rheumatoid arthritis: focus on early disease. Ann Rheum Dis 2016;75:1268-71.

14 Verschueren P, De Cock D, Corluy L, et al. Patients lacking classical poor prognostic markers might also benefit from a step-down glucocorticoid bridging scheme in early rheumatoid arthritis: week 16 results from the randomized multicenter CareRA trial. Arthritis Res Ther 2015;17:97.

15 van den Broek M, Dirven L, Klarenbeek NB, et al. The association of treatment response and joint damage with ACPA-status in recent-onset RA: a subanalysis of the 8-year follow-up of the BeSt study. Ann Rheum Dis 2012;71:245-8.

16 van Nies JA, Tsonaka R, Gaujoux-Viala C, et al. Evaluating relationships between symptom duration and persistence of rheumatoid arthritis: does a window of opportunity exist? Results on the Leiden early arthritis clinic and ESPOIR cohorts. Ann Rheum Dis 2015;74:806-12.
17 van Nies JA, Krabben A, Schoones JW, et al. What is the evidence for the presence of a therapeutic window of opportunity in rheumatoid arthritis? A systematic literature review. Ann Rheum Dis 2014;73:861-70.

18 de Hair MJ, Lehmann KA, van de Sande MG, et al. The clinical picture of rheumatoid arthritis according to the 2010 American College of Rheumatology/European League Against Rheumatism criteria: is this still the same disease? Arthritis Rheum 2012:64:389-93.

19 Ferraccioli G, Tolusso B, Fedele AL, et al. Do we need to apply a T2T strategy even in ACPA-negative early rheumatoid arthritis? YES. RMD Open 2016;2: e000263.

20 De Winter LM, Hansen WL, van Steenbergen HW, et al. Autoantibodies to two novel peptides in seronegative and early rheumatoid arthritis. Rheumatology (Oxford) 2016;55:1431-6.

21 Shi J, Knevel R, Suwannalai $P$, et al. Autoantibodies recognizing carbamylated proteins are present in sera of patients with rheumatoid arthritis and predict joint damage. Proc Natl Acad Sci USA 2011;108: 17372-7.

22 Juarez $\mathrm{M}$, Bang $\mathrm{H}$, Hammar $\mathrm{F}$, et al. Identification of novel antiacetylated vimentin antibodies in patients with early inflammatory arthritis. Ann Rheum Dis 2016;75:1099-107.

23 Navalho $\mathrm{M}$, Resende C, Rodrigues AM, et al. Bilateral evaluation of the hand and wrist in untreated early inflammatory arthritis: a comparative study of ultrasonography and magnetic resonance imaging. J Rheumatol 2013;40:1282-92.

24 Nieuwenhuis WP, van Steenbergen HW, Mangnus L, et al. Diagnostic accuracy of hand and foot MRI for early rheumatoid arthritis. [abstract]. 2016. doi:10.1136/annrheumdis-2016-eular.2723

25 Minowa K, Ogasawara M, Murayama G, et al. Predictive grade of ultrasound synovitis for diagnosing rheumatoid arthritis in clinical practice and the possible difference between patients with and without seropositivity. Mod Rheumatol 2016;26:188-93. 